\title{
Interplay between crystal-size and disorder effects in the high-energy optical response of photonic crystal slabs
}

\author{
Luis A. Dorado and Ricardo A. Depine* \\ Grupo de Electromagnetismo Aplicado, Departamento de Física, Facultad de Ciencias Exactas y Naturales, Universidad de Buenos \\ Aires, Buenos Aires C1428EGA, Argentina \\ Gabriel Lozano and Hernán Míguez \\ Instituto de Ciencia de Materiales de Sevilla, Consejo Superior de Investigaciones Científicas, Sevilla 41092, Spain
}

(Received 20 June 2007; revised manuscript received 13 August 2007; published 5 December 2007)

\begin{abstract}
Experimental reflectance spectra have been obtained for colloidal crystals whose widths ranged from one to several sphere monolayers, and their features in the higher order band energy range have been reproduced theoretically. In order to fit the measured data, optical extinction has been introduced in the theoretical model, which accounts for structure imperfections and disorder, the main sources of losses in an actual measurement. A complex spectrum in the high frequency region is observed even for one ordered monolayer, being this peak structure gradually modified as more layers are piled up. This allowed us to identify which peaks are reminiscent of the optical reflectance features of a single close-packed layer and which are the result of building up a three dimensional periodicity. A clear correlation between the amount of extinction introduced in the fitting and the slab width has been found, which demonstrates that wider real crystals produce less diffusely scattered light. At the same time, we find that the optical response of thinner crystals is more robust against the introduction of extinction than that of thicker ones, for which the effect is dramatic.
\end{abstract}

DOI: 10.1103/PhysRevB.76.245103

PACS number(s): 78.20.Bh, 78.40.-q, 42.70.Qs, 41.20.Jb

Improvements in the processes of fabrication of selfassembled three dimensional (3D) photonic crystals ( $\mathrm{PhCs}$ ), which are materials with a spatially periodic dielectric function in all three dimensions, have made possible the observation of high quality optical spectra in higher order band frequencies. ${ }^{1-3}$ Also, the appearance of localized modes inside band gaps in the photonic band structure due to the addition of local defects is a well-known effect, which is analogous to the doping of semiconductors. ${ }^{4}$ Point, line, or plane defects are created within the crystal by locally adding or removing material in a controlled manner, ${ }^{5,6}$ so they are different from intrinsic defects, which are unintentional disruptions of the spatial periodicity of the dielectric function that arise during the fabrication process. Despite new fabrication techniques that have alleviated the effect of disorder in the so-called low-energy range, where the lattice constant is less than the wavelength of light, very recently, it has been demonstrated that extinction due to intrinsic defects in $3 \mathrm{D}$ PhCs strongly affects the shape of the experimental spectra in the high-energy range, where the lattice constant is greater than the wavelength. ${ }^{7}$ Also, calculations of the photonic band structure, performed considering extinction, have shown a clear correlation between the behavior of the imaginary part of the wave vector and the optical features observed in the spectra. This means that disorder, while having a small influence on the measured spectra in the low-energy range, amplifies its effects at higher energies and, consequently, further improvement of the fabrication techniques is required to achieve optical quality at those photon energies. ${ }^{8}$

In order to perform a valid comparison between the optical spectra of finite crystal slabs and the photonic band structure of an infinite crystal, $\mathrm{PhC}$ slabs of several layers were analyzed in a previous work; an excellent agreement between the experimental and the calculated optical response at high energies has been found. Here, we present an analysis of the behavior of the optical spectra features in the high-energy range as the width of the crystal slab is gradually increased. We compare the experimental and theoretical evolutions of the specular reflectance spectra as we increase the crystal thickness from 1 to 18 sphere layers. We found complex spectra in the high frequency region even for the initial monolayer structure, ${ }^{9}$ being this peak structure gradually modified as more layers are piled up. This allowed us to identify which peaks are reminiscent of the optical reflectance features of a single close-packed layer and which are the result of building up a three dimensional periodicity. At the same time, we find a clear correlation between the crystal size and the amount of extinction needed to attain the best fit. The interplay of crystal size and disorder effects on the highenergy optical response is thoroughly analyzed, finding a much more dramatic effect of the former on the reflectance spectra of larger crystals.

Photonic crystal films were made by evaporation induced self-assembly ${ }^{10}$ on glass substrates of polystyrene sphere (IKERLAT, average diameter of $700 \mathrm{~nm}$, polydispersity below $3 \%$, density $\rho=1.1 \mathrm{~g} / \mathrm{cm}^{3}$, refractive index $\left.n=1.59\right)$ suspended in water with particle volume fraction ranging from $0.05 \%$ to $0.20 \%$ and evaporated at temperatures ranging from 30 to $60{ }^{\circ} \mathrm{C}$. Several independent measurements of the high-energy optical response of similar structures can be found in the literature. ${ }^{1,11,12}$ Thus, the technique is sufficiently mature and reliable to provide reproducible results in different laboratories. The typical crystal growth direction is the [111], and we will focus on the optical properties when light impinges in that particular direction since they are best known. ${ }^{13}$ Thus, in this case, a monolayer consists of a triangular lattice of close-packed spheres and the slab width is increased by piling monolayers up in the sequence 
$A B C A B C \cdots$. As it is usual, the photon energy is expressed in reduced units $a / \lambda$, where $a$ is the lattice constant of the classical cubic cell, whose value is $a=\sqrt{2} \phi$ in an fcc closepacked structure, $\phi$ being the sphere diameter and $\lambda$ the wavelength of the incident light.

Reflectance measurements were performed using a Fourier transform infrared spectrophotometer (BRUKER IFS66) attached to a microscope. A $4 \times$ objective with a numerical aperture of 0.1 (light cone angle of $\pm 5.7^{\circ}$ ) was used to irradiate the lattices and to collect the reflected light at quasinormal incidence with respect to its surface. A spatial filter was used to selectively detect light from square spots of $1000 \times 1000 \mu \mathrm{m}^{2}$.

Simulated reflectance spectra were calculated by means of the vector Korringa-Kohn-Rostoker method. ${ }^{14,15}$ To ensure convergence in the high-energy range, we used 41 two dimensional (2D) reciprocal lattice vectors in the plane wave expansions and spherical waves with angular momentum up to $l_{\max }=9$. These values are the minimum ones that provide good convergence in that range. Lower values of $l_{\max }$ and less reciprocal lattice vectors still give a correct result for lower-energy bands, but it is not sufficient to provide trustful information on the region we are interested in. Measurements were performed with the $\mathrm{PhC}$ slab deposited on a glass substrate of refractive index 1.53 , so this substrate is also included in the theoretical model. The incoming medium is air (refractive index $=1$ ). The spheres are embedded in air and have a complex dielectric constant $\varepsilon_{s}=2.5+\underline{i \varepsilon_{i}}$, which corresponds to latex spheres (refractive index $=\sqrt{2.5}$ $\cong 1.58$ ). An imaginary part $\varepsilon_{i}$ is added to the dielectric constant of the spheres in order to take into account all possible sources of losses in actual measurements. Then, the value of $\varepsilon_{i}$ is chosen to fit the experimental data.

Energy losses due to power dissipation are negligible in latex spheres, so structure imperfections and disorder are responsible for the experimental deviation of the spectrum shape with respect to the theoretical optical response without extinction. ${ }^{7}$ In the high-energy range, the average size of these structural imperfections, measured in wavelengths, becomes more important than that in the low-energy bands. For this reason, these intrinsic defects ${ }^{16}$ cause light to be scattered diffusely, removing part of the energy from the specularly reflected or ballistically transmitted beam. In this work, we will focus on the analysis of specular reflectance results, since they can be readily attained by microspectroscopy from single domains of the crystalline film using a low numerical aperture objective, ensuring the thickness uniformity of the tested spot and the normal incidence of the beam. This is a much more complicated task to be performed in transmission mode, since the incident beam is typically focused with a larger numerical aperture objective.

In Figs. 1 and 2, the measured and calculated specular reflectances ( or $R_{0,0}$ ) can be seen as a function of the lattice constant $a / \lambda$ for different crystal slab widths. Very good agreement between the experimental and theoretical reflectance spectra has been obtained by introducing extinction in the theoretical model, as we have demonstrated for much thicker crystals before. Figure 1 shows the experimental and theoretical specular reflectivity spectra for PhC slabs of a few layers $\left(N=\right.$ number of layers). The value of $\varepsilon_{i}$ in the

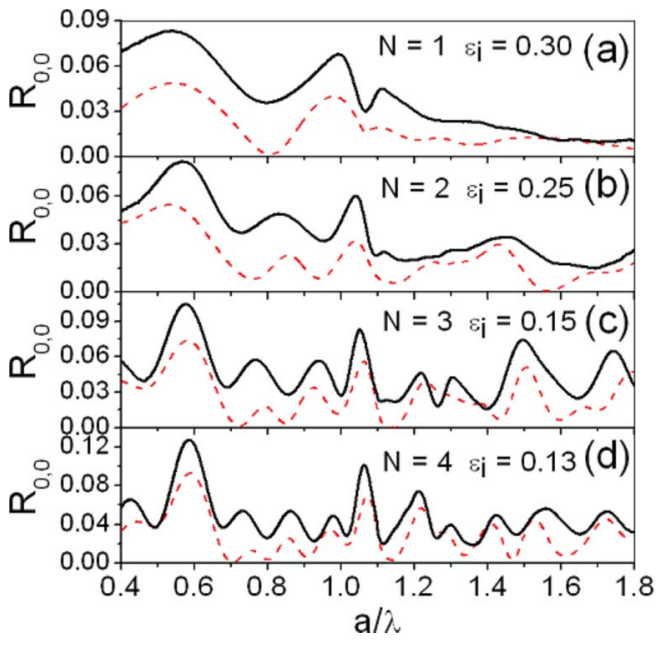

FIG. 1. (Color online) Measured (solid lines) and calculated (dashed lines) specular reflectance spectra for glass-supported slabs of $N$ layers composed of spheres of dielectric constant $\varepsilon_{s}=2.5+i \varepsilon_{i}$ in air. (a) $N=1, \varepsilon_{i}=0.30$. (b) $N=2, \varepsilon_{i}=0.25$. (c) $N=3, \varepsilon_{i}=0.15$. (d) $N=4, \varepsilon_{i}=0.13$.

calculated spectra was chosen to obtain the best fit. Note that all oscillations present a similar height with respect to the background in both the experimental (black solid lines) and the simulated (red dashed lines) spectra, which was actually the criterion followed to choose the best fit. Differences in the baseline are due to the fact that only one interface of the glass substrate is considered for the calculations in order to avoid the presence of very short frequency lobes in the spectra. These arise from the interference between beams reflected at the upper and lower faces of the thick substrate $(1 \mathrm{~mm})$ under consideration. Also, some dispersion in the real part of the dielectric constant was added, which accounts for a small refractive index increase of materials as we move toward higher energies.

In Fig. 1(a), two main peaks for the monolayer $(N=1)$ can

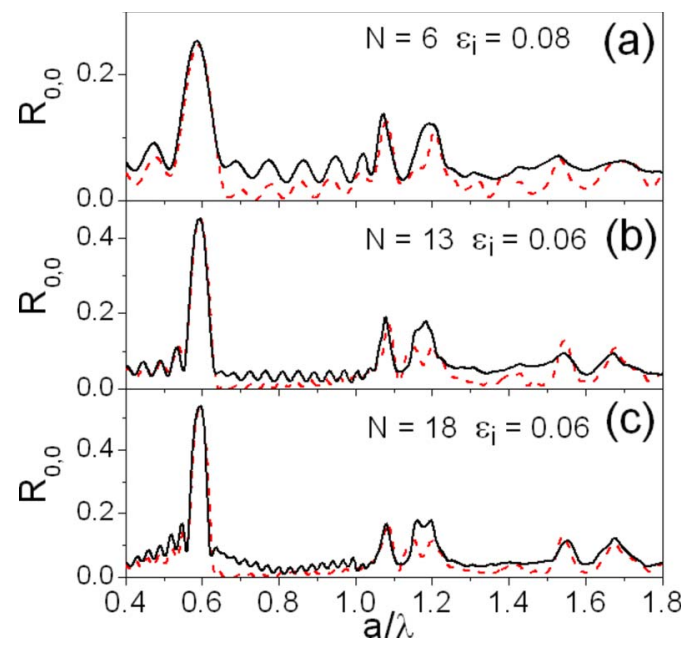

FIG. 2. (Color online) Measured (solid lines) and calculated (dashed lines) specular reflectance spectra for glass-supported slabs of $N$ layers composed of spheres of dielectric constant $\varepsilon_{s}=2.5+i \varepsilon_{i}$ in air. (a) $N=6, \varepsilon_{i}=0.08$. (b) $N=13, \varepsilon_{i}=0.06$. (c) $N=18, \varepsilon_{i}=0.06$. 
be seen: one near $a / \lambda=0.55$ and the other at $a / \lambda=1.0$. These peaks move gradually toward higher energies as the slab width is increased, as Figs. 1(a)-1(c) show. Also, secondary peaks related to the Fabry-Perot oscillations appear between these main peaks for slabs of $N=2,3$, and 4 layers. Since all our measurements were taken far from the edges of the crystal, in which different types of stacking sequences have been reported, ${ }^{17}$ the fairness of the fittings we attain considering only the $A B C A B C \cdots$ sequence indicates that an fcc lattice is built as more monolayers are piled up.

In Fig. 2, the reflectivity spectra for PhC slabs of $N=6$, 13, and 18 layers can be appreciated. The main peak in the low-energy band tends to its final position, which is at $a / \lambda$ $=0.61$, as can be predicted from the photonic band structure of the sphere fcc lattice under consideration, because there is a pseudoband gap in the $\Gamma L$ direction centered at this particular energy. ${ }^{7}$ The first peak in the high-energy region has moved to $a / \lambda=1.07$, and a second double degenerated peak near $a / \lambda=1.2$ is present. It is interesting to note that this second peak becomes double degenerated in slabs with at least six layers. In other words, we must complete at least two $A B C$ sequences in the slab in order to obtain the peak at $a / \lambda=1.2$. Figure 2 also shows that this peak is difficult to see in the measured data for $N=6$ and 13 layers, but Fig. 2(c) for $N=18$ layers confirms its existence. There is a second peak structure in the range of $1.5<a / \lambda<1.8$, which is composed of two peaks of similar amplitudes. They can be observed in Figs. 1(c), 1(d), and 2, so the number of layers needed must be $N \geq 3$, which corresponds to one or more complete sequences of $A B C$. The gradual appearance of these highenergy peaks as the number of sphere layers is increased can be predicted from the photonic band structure calculated with extinction. ${ }^{7}$ However, for $a / \lambda>1.63$, diffracted spots remove part of the energy from the specularly reflected beam. Then, above that energy, both disorder and diffraction contribute to the reduction of intensity of the specularly reflected light.

The ratio between the power carried by each diffracted mode and the incident power is given by a reflection coefficient $R_{p, q}$, where the pair of integers $(p, q)$ indicate the order of diffraction, and $(p, q)=(0,0)$ corresponds to the specularly reflected light, the quantity that has been measured for this work. The onset of diffraction in air at $a / \lambda=1.63$ can be predicted by requiring the conservation of the tangential component of the wave vectors for a $2 \mathrm{D}$ triangular lattice, that is,

$$
\frac{a}{\lambda} \geqslant \frac{\sqrt{2}}{n} \sqrt{p^{2}+\frac{(2 q+p)^{2}}{3}}
$$

where $n$ is the refractive index of the diffraction medium ( $n=1$ for air). This same expression explains the appearance of diffracted modes only within the glass substrate $(n=1.53)$ and not in the incoming medium at $a / \lambda=1.07$ for the supported lattice. ${ }^{7}$ In Fig. 3, we compare the calculated spectrum of $R_{0,0}$ and that of $\Sigma_{(p, q) \neq(0,0)} R_{p, q}$ (the sum of the intensities of all diffracted modes in the homogeneous incident medium of refractive index $n=1$ ) for the slab composed of 18 layers. The total reflectivity $R=\Sigma_{(p, q)} R_{p, q}$ is also plotted

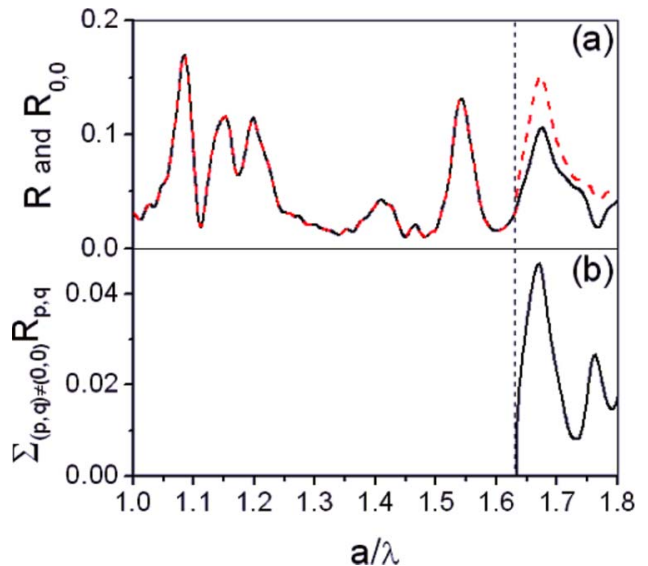

FIG. 3. (Color online) (a) Calculated specular (solid line) and total (dashed line) reflectance spectra, $R_{0,0}$ and $R$, for a glasssupported slab of 18 layers and spheres of dielectric constant $\varepsilon_{s}$ $=2.4964+0.06 i$ in air. (b) Reflected diffracted light, $\Sigma_{(p, q) \neq(0,0)} R_{p, q}$, spectra. The vertical dashed line indicates the onset of diffraction.

in Fig. 3(a), where the intensity reduction of $R_{0,0}$ compared to $R$ can be appreciated for photon energies $a / \lambda>1.63$.

The impact of disorder in the optical response of the glass-supported $\mathrm{PhC}$ slab can be observed in Fig. 4, where the specular reflectance spectra for $N=1$ and 5 layers is shown for different values of the imaginary dielectric constant $\varepsilon_{i}$. The attenuation of the reflectance values compared to the case $\varepsilon_{i}=0.0001$, which corresponds to a slab with almost no disorder, can clearly be seen in both cases. However, this effect is much more dramatic in the case of the five layer lattices, whose maximum at $a / \lambda=1.15$ is decreased by a factor above 10, while for the monolayer, the corresponding maximum at $a / \lambda=1.0$ is reduced by factor smaller than 2 . This is a theoretical confirmation that the thinner the crystals,

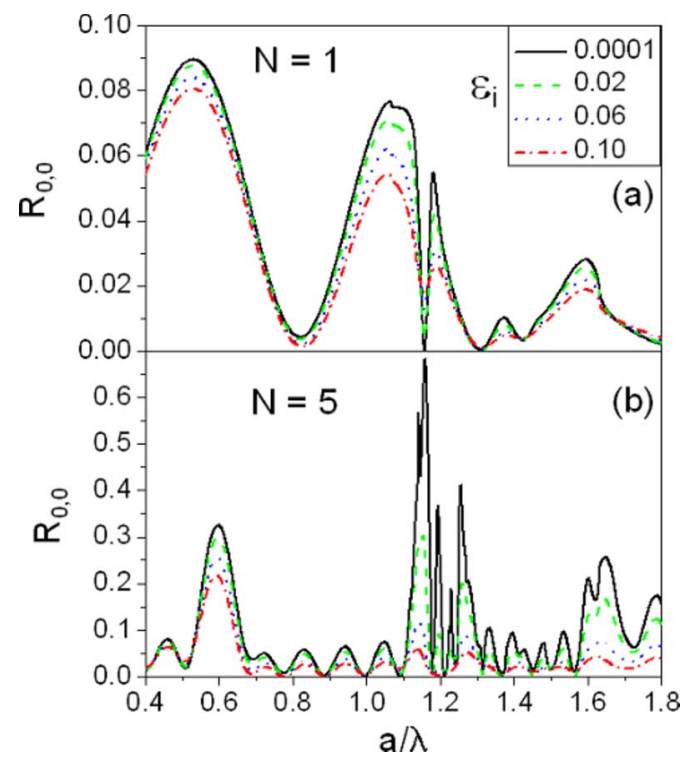

FIG. 4. (Color online) Calculated specular reflectance spectra for (a) a glass-supported monolayer $(N=1)$ and (b) a glasssupported slab of $N=5$ layers, both with spheres of dielectric constant $\varepsilon_{s}=2.5+i \varepsilon_{i}$ in air for different values of $\varepsilon_{i}$. 


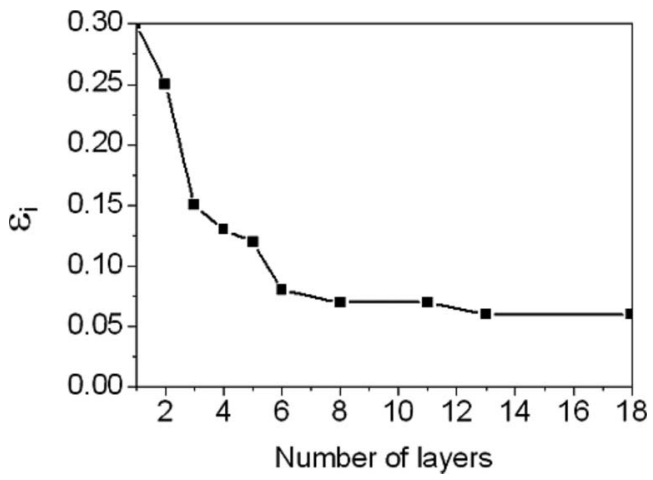

FIG. 5. Imaginary part of the dielectric constant of the spheres as a function of the number of layers of the $\mathrm{PhC}$ slab.

the more robust its optical response against the introduction of extinction.

In the experimental spectra of Figs. 1 and 2, we can appreciate a gradual increase of the reflectance amplitudes as the number of layers is increased. Also, the imaginary part $\varepsilon_{i}$ of the dielectric constant of the spheres decreases as the number of layers $N$ is increased in the calculated spectra. This tendency is plotted in Fig. 5, where $\varepsilon_{i}$ can be seen as a function of $N$. Hence, there exists a clear correlation between the extinction introduced and the width of the slab, which indicates that diffusely scattered light increases for thinner crystals. In the case of crystals of a few layers, besides the effect of disorder, interactions with the glass substrate produce an additional attenuation of the reflectivity values. Therefore, the increase of $\varepsilon_{i}$ as the crystal width is decreased means that more diffusely scattered light is produced, the combination of disorder and glass-crystal interaction being responsible of this effect. Nevertheless, for crystals with six or more layers, $\varepsilon_{i}$ becomes practically a constant, as Fig. 5 shows, which means that any surface effect and glass-crystal interaction are negligible, the value of $\varepsilon_{i}$ being a measure of the degree of disorder in these cases. Thus, we found a method for quantifying disorder in a real crystal because we can use a single parameter such as $\varepsilon_{i}$, which is determined by fitting the theoretical model to the experimental data, as a measure of the structural quality of the PhC slab. A similar method has already been proposed for the same type of $\mathrm{PhC}$ films but based on the fitting of the low-energy optical response of the lattices. ${ }^{18}$ Interestingly, the same trend of the extinction needed to fit the experimental curve versus the number of close-packed layers in the film is found.

In conclusion, we have realized an integral approach to the optical response of 3D PhC slabs as a function of the slab width in the higher order band energy range. By analyzing the gradual modification of that response from one to several monolayers, we could identify which peaks are reminiscent of the optical reflectance features of a single close-packed layer and which are the result of building up a three dimensional periodicity. Furthermore, we have found a clear correlation between the extinction introduced in our theoretical model and the width of the real slab, which indicates that wider crystal slabs produce less diffusely scattered light and provides a method for quantifying disorder in these cases.

This work has been realized in the framework of a joint Spanish-Argentinian cooperation project CSIC-CONICET (Grant No. 2005AR0070). R.A.D. and L.A.D. acknowledge support from Consejo Nacional de Investigaciones Científicas y Técnicas (CONICET), Universidad de Buenos Aires (UBA), and Agencia Nacional de Promoción Científica y Tecnológica (ANPCYT-BID 802/OC-AR03-14099). H.M. is grateful for financial support from the Ramón Areces Foundation and the Spanish Ministry of Science and Education under Grant No. MAT2005-03028. *rdep@df.uba.ar

${ }^{1}$ H. Míguez, V. Kitaev, and G. Ozin, Appl. Phys. Lett. 84, 1239 (2004).

${ }^{2}$ F. García-Santamaría, J. F. Galisteo-López, P. V. Braun, and C. López, Phys. Rev. B 71, 195112 (2005).

${ }^{3}$ K. Wostyn, Y. Zhao, B. Yee, K. Clays, A. Persoons, G. de Schaetzen, and L. Hellemans, J. Chem. Phys. 118, 10752 (2003).

${ }^{4}$ E. Yablonovitch, T. J. Gmitter, R. D. Meade, A. M. Rappe, K. D. Brommer, and J. D. Joannopoulos, Phys. Rev. Lett. 67, 3380 (1991).

${ }^{5}$ P. V. Braun, S. A. Rinne, and F. Garcia-Santamaria, Adv. Mater. (Weinheim, Ger.) 18, 2665 (2006).

${ }^{6}$ A. Arsenault, F. Fleischhaker, G. von Freymann, V. Kitaev, H. Míguez, A. Mihi, N. Tetreault, E. Vekris, I. Manners, S. Aitchison, D. Perovic, and G. A. Ozin, Adv. Mater. (Weinheim, Ger.) 18, 2779 (2006).

${ }^{7}$ L. A. Dorado, R. A. Depine, and H. Míguez, Phys. Rev. B 75, 241101(R) (2007).

${ }^{8}$ Z. Y. Li and Z. Q. Zhang, Phys. Rev. B 62, 1516 (2000).

${ }^{9}$ S. Yano, Y. Segawa, J. S. Bae, K. Mizuno, S. Yamaguchi, and K.
Ohtaka, Phys. Rev. B 66, 075119 (2002).

${ }^{10}$ J. F. Bertone, P. Jiang, K. S. Hwang, D. M. Mittleman, and V. L. Colvin, Phys. Rev. Lett. 83, 300 (1999).

${ }^{11}$ J. F. Galisteo-López and C. López, Phys. Rev. B 70, 035108 (2004).

${ }^{12}$ S. Wong, V. Kitaev, and G. A. Ozin, J. Am. Chem. Soc. 125, 15589 (2003).

${ }^{13}$ P. Jiang, J. F. Bertone, K. S. Hwang, and V. L. Colvin, Chem. Mater. 11, 2132 (1999).

${ }^{14}$ N. Stefanou, V. Yannopapas, and A. Modinos, Comput. Phys. Commun. 113, 4977 (1998); 132, 189 (2000).

${ }^{15}$ N. Stefanou, V. Karathanos, and A. Modinos, J. Phys.: Condens. Matter 4, 7389 (1992).

${ }^{16}$ E. Lidorikis, M. M. Sigalas, E. N. Economou, and C. M. Soukoulis, Phys. Rev. Lett. 81, 1405 (1998).

${ }^{17}$ X. Checoury, S. Enoch, C. López, and A. Blanco, Appl. Phys. Lett. 90, 161131 (2007).

${ }^{18}$ J. F. Galisteo-López, M. Galli, M. Patrini, A. Balestreri, L. C. Andreani, and C. López, Phys. Rev. B 73, 125103 (2006). 